\title{
Short communication: Genotype imputation within and across Nordic cattle breeds
}

\author{
R. F. Brøndum, ${ }^{* 1}$ P. Ma, ${ }^{\star} \dagger$ M. S. Lund, ${ }^{*}$ and G. $S u^{\star 1}$ \\ ${ }^{*}$ Centre for Quantitative Genetics and Genomics, Department of Molecular Biology and Genetics, Aarhus University, DK-8830 Tjele, Denmark \\ †Department of Animal Genetics, College of Animal Science and Technology, China Agricultural University, Beijing 100193, P. R. China
}

\begin{abstract}
This study investigated the accuracies of imputation from $50 \mathrm{~K}$ genotypes to high-density genotypes for animals from the Danish, Swedish, or Finnish Red dairy cattle populations, using either a national, combined Red, or combined Red and Holstein reference population. Combining the Red populations increased the imputation accuracy for all 3 populations compared with using single-nationality references. Including Holstein animals in the reference further increased the imputation accuracy for Danish Red.
\end{abstract}

Key words: genotype imputation, combined reference population

\section{Short Communication}

The new high-density (HD) SNP marker panel (777K) from Illumina Inc. (San Diego, CA) is expected to yield higher reliabilities in genomic prediction and higher precision in mapping of QTL than the lower density SNP marker panel. Because it is not economically feasible to genotype all animals of interest with an HD marker panel, an efficient approach to obtain HD marker data is to extend lower density marker data to HD marker data by using statistical models to impute the missing markers. With imputed data, a risk of errors exists, and these errors are known to reduce the power in genome-wide association studies (Huang et al., 2009b) and the reliability of genomic predictions (Weigel et al., 2010; Dassonneville et al., 2011a; Mulder et al., 2012). Therefore, the accuracy of the imputation method is an important issue in genome-wide association studies and genomic predictions. Accurate imputation depends not only on the statistical model, but also on the strategy for using the available data. Previous results have shown that

Received March 30, 2012

Accepted July 6, 2012

${ }^{1}$ Corresponding authors: rasmusf.brondum@agrsci.dk and guosheng. su@agrsci.dk the accuracy is higher when the imputations are done within breed than across breeds (Hayes et al., 2012). These results were, however, obtained using data from an SNP marker panel with lower marker density (imputation from $5 \mathrm{~K}$ to $50 \mathrm{~K}$ in sheep), for which the correlation of the linkage disequilibrium (LD) phase between breeds might be low. For the new HD chip, however, the marker density is 14-fold higher than that of the previous $50 \mathrm{~K}$ chip, which means that the correlation of the LD phase is much higher even across breeds. For example, the correlation of the pairwise LD of adjacent markers between Nordic Holstein and Nordic Red dairy cattle (RDC) increases from 0.782 for markers in the $50 \mathrm{~K}$ chip to 0.967 in the $777 \mathrm{~K}$ chip (G. Su, unpublished data). A previous study showed that higher imputation accuracies were obtained in most human populations when a mixture of HapMap reference panels was used compared with a single Hapmap reference, when imputing missing markers on an SNP marker panel at a density slightly lower than that of the BovineHD (15\% missing in 500K; Huang et al., 2009a).

In a previous study on the reliability of genomic predictions in the RDC populations, the reliability has been shown to increase when using pooled reference data from the 3 Red populations (Brøndum et al., 2011). The reliability of genomic predictions across populations is highly affected by the LD and persistence of the LD phase between populations. The same holds true for the accuracy of imputation, and it can therefore be expected that the accuracy of imputed markers in the $\mathrm{RDC}$ populations also will increase when the genotype data from the 3 populations are pooled.

Previous attempts to combine reference populations from RDC with Holstein cattle for genomic prediction showed a slight increase in the reliability of genomic prediction for some traits (G. Su, unpublished data). Because some Holstein bulls have previously been used in the breeding program for Danish Red cattle, including Holstein bulls in the reference for imputation might offer improved accuracy. The purpose of this study was to investigate the effect of combining reference populations when imputing markers from a $50 \mathrm{~K}$ marker panel to an HD marker panel for RDC and Holstein cattle. 


\section{Data}

The HD genotype data obtained using the Illumina 777K BovineHD genotype chip was available for 706 RDC bulls (247 Danish, 210 Swedish, and 249 Finnish) and 546 Holstein bulls from the Eurogenomics collaboration (Lund et al., 2011). Quality control consisted of removing markers with a call frequency less than 0.95, monomorphic markers, and markers without a map position. After quality control, 612,615 SNP on chromosomes 1 to 29 remained for analysis in the RDC populations, and 605,728 remained for the Holsteins.

\section{Methods}

To investigate whether a combined multipopulation (RDC) or multibreed (RDC + Holstein) reference data set could increase the imputation accuracy, each of the $\mathrm{RDC}$ populations was imputed by using either national reference data or the combined RDC data. A combined Swedish-Finnish reference was also attempted because the genetic links between these 2 populations are stronger than the genetic links between them and the Danish Red (Brøndum et al., 2011). Finally, imputation using a multibreed reference including both RDC and European Holsteins was investigated.

Fifty bulls from each of the Danish, Swedish, and Finish populations and 100 Holstein bulls were randomly selected for validation, with the requirement that they did not have sons in the reference. For validation bulls, markers in the HD data that were not on the $50 \mathrm{~K}$ chip were deleted. This also removed approximately 4,000 markers from the $50 \mathrm{~K}$ chip that were not included on the HD chip. The procedure left 39,239 markers in the validation set for the RDC populations and 42,107 markers for the Holstein validation animals. The deleted markers were then imputed using Beagle software v. 3.3 (Browning and Browning, 2009). Beagle was run with the default scale and shift parameters; no information on the relationships between individuals was provided, and the outputs used for further analysis were the most probable genotypes.

Imputation accuracy was measured by the mean allele error rates (i.e., the number of falsely imputed alleles divided by the total number of imputed alleles) and by the mean correlation of true and imputed genotypes. Allele error rates were averaged across all loci and animals within each validation population, and correlations were calculated at each locus for each validation population and then averaged across loci. Markers that were missing in the true genotype data were disregarded when calculating the error rates and correlations.

\section{Results and Discussion}

Table 1 shows the imputation accuracies for the 3 RDC populations when using different combinations of the 3 as references. The results clearly demonstrated that using the full RDC reference was superior to using the single-nationality references. The gain was larger for the Danish and Swedish animals than for the Finnish animals, and compared with using only a Swedish-Finnish reference, the Finnish animals did not achieve any extra gain by including Danish animals in the reference. The patterns were similar to those of the genomic predictions in Brøndum et al. (2011), in which using a combined RDC reference population yielded an improvement in the reliability of genomic predictions for all 3 RDC populations compared with using singlenationality reference populations, and the reliabilities of Swedish and Finnish animals did not increase by including Danish animals in the reference, compared with using a Swedish-Finnish reference. These similar patterns of imputation accuracy and reliability of genomic prediction were likely due to the stronger genetic links between the Finnish and Swedish RDC populations and the weaker links between these 2 populations and

Table 1. Mean allele error rate and standard deviation (in parentheses) across animals, and mean correlation between true and imputed genotypes (R) when using single- or multipopulation references for Danish Red (RDM), Swedish Red (SRB), Finnish Ayrshire (FAY), and all Nordic Red (RDC) animals

\begin{tabular}{lllccc}
\hline Reference & $\begin{array}{c}\text { Reference } \\
\text { (no.) }\end{array}$ & Validation & $\begin{array}{c}\text { Validation } \\
\text { (no.) }\end{array}$ & Error rate & $\mathrm{R}$ \\
\hline RDM & 197 & RDM & 50 & $2.64(2.43)$ & 0.925 \\
SRB & 160 & SRB & 50 & $1.39(1.42)$ & 0.958 \\
FAY & 199 & FAY & 50 & $0.87(0.58)$ & 0.973 \\
RDC & 556 & RDM & 50 & $1.75(1.84)$ & 0.950 \\
& & SRB & 50 & $0.59(0.29)$ & 0.982 \\
& & FAY & 50 & $0.54(0.30)$ & 0.983 \\
SRB + FAY & RDC & 150 & $0.96(1.22)$ & 0.971 \\
& \multirow{2}{*}{359} & SRB & 50 & $0.96(1.31)$ & 0.971 \\
& & FAY & 50 & $0.54(0.31)$ & 0.983 \\
& & SRB + FAY & 100 & $0.75(0.97)$ & 0.976 \\
\hline
\end{tabular}


Table 3. Mean allele error rate and standard deviation (in parentheses) across animals, and mean correlation between true and imputed genotypes (R) for Holstein (HOL), Danish Red (RDM), Swedish Red (SRB), Finnish Ayrshire (FAY), and all Nordic Red (RDC) animals using a single- or multibreed reference

\begin{tabular}{|c|c|c|c|c|c|}
\hline Reference & $\begin{array}{l}\text { Reference } \\
\text { (no.) }\end{array}$ & Test & $\begin{array}{l}\text { Validation } \\
\quad \text { (no.) }\end{array}$ & Error rate & $\mathrm{R}$ \\
\hline $\mathrm{HOL}$ & 457 & HOL & 100 & $0.77(0.61)$ & 0.975 \\
\hline \multirow[t]{4}{*}{$\mathrm{RDC}$} & 556 & RDM & 50 & $1.75(1.84)$ & 0.950 \\
\hline & & SRB & 50 & $0.59(0.29)$ & 0.982 \\
\hline & & FAY & 50 & $0.54(0.30)$ & 0.983 \\
\hline & & $\mathrm{RDC}$ & 150 & $0.96(1.22)$ & 0.971 \\
\hline \multirow[t]{5}{*}{$\mathrm{HOL}+\mathrm{RDC}$} & 1,013 & HOL & 100 & $0.68(0.52)$ & 0.977 \\
\hline & & RDM & 50 & $1.17(1.35)$ & 0.967 \\
\hline & & SRB & 50 & $0.60(0.29)$ & 0.982 \\
\hline & & FAY & 50 & $0.55(0.28)$ & 0.983 \\
\hline & & $\mathrm{RDC}$ & 150 & $0.77(0.85)$ & 0.977 \\
\hline
\end{tabular}

at each imputed locus on chromosome 1 when using a reference of only RDC animals compared with a reference of RDC and Holstein animals. It can be seen that with the multibreed reference, a tendency toward lower error rates existed for most loci, but some loci did have higher error rates (left panel of Figure 4). To inves-
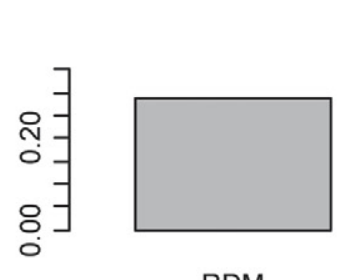

RDM
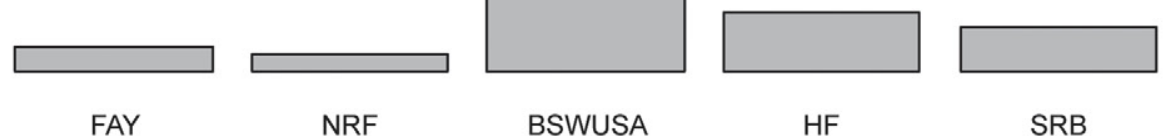

Breeds with mean proportion $>1 \%$
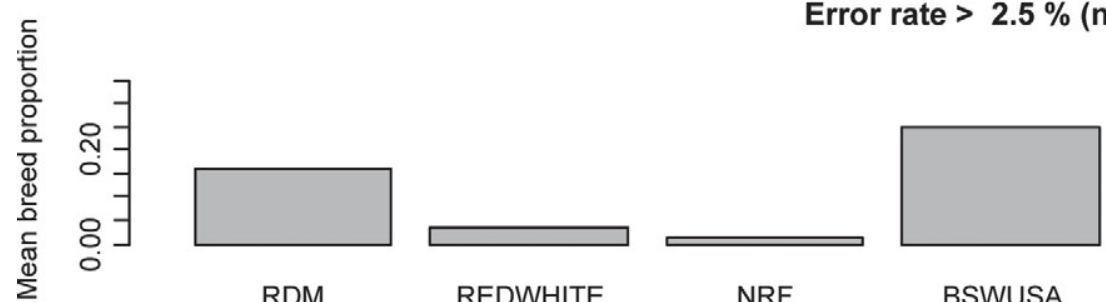

RDM
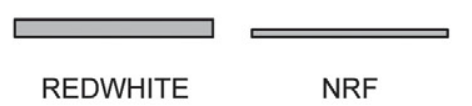

BSWUSA

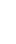

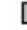

Breeds with mean proportion $>1 \%$

Reference animals $(n=553)$
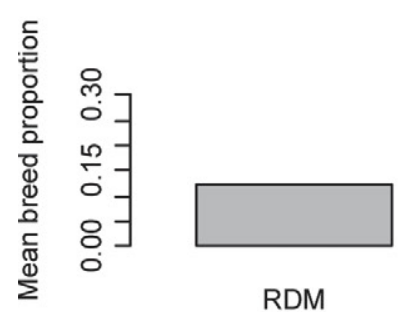

RDM

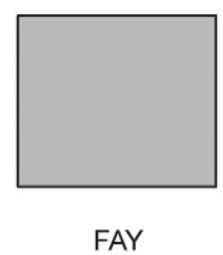

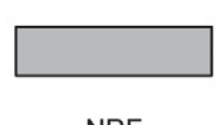

NRF

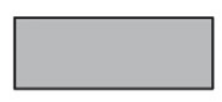

BSWUSA

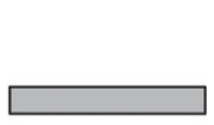

HF

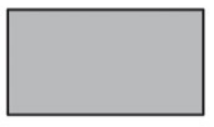

SRB

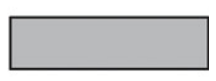

ANGLER

Breeds with mean proportion $>1 \%$

Figure 2. Mean breed proportions for Danish Red animals with an error rate above or below $2.5 \%$ and mean breed proportions for the Nordic Red reference animals. Breeds are abbreviated as follows: Danish Red (RDM), Finnish Ayrshires (FAY), Swedish Red (SRB), Norwegian Red (NRF), American Brown Swiss (BSWUSA), Holstein-Friesian (HF), Red Holstein (REDWHITE), Canadian Ayrshires (CAY), and German Red (ANGLER). 


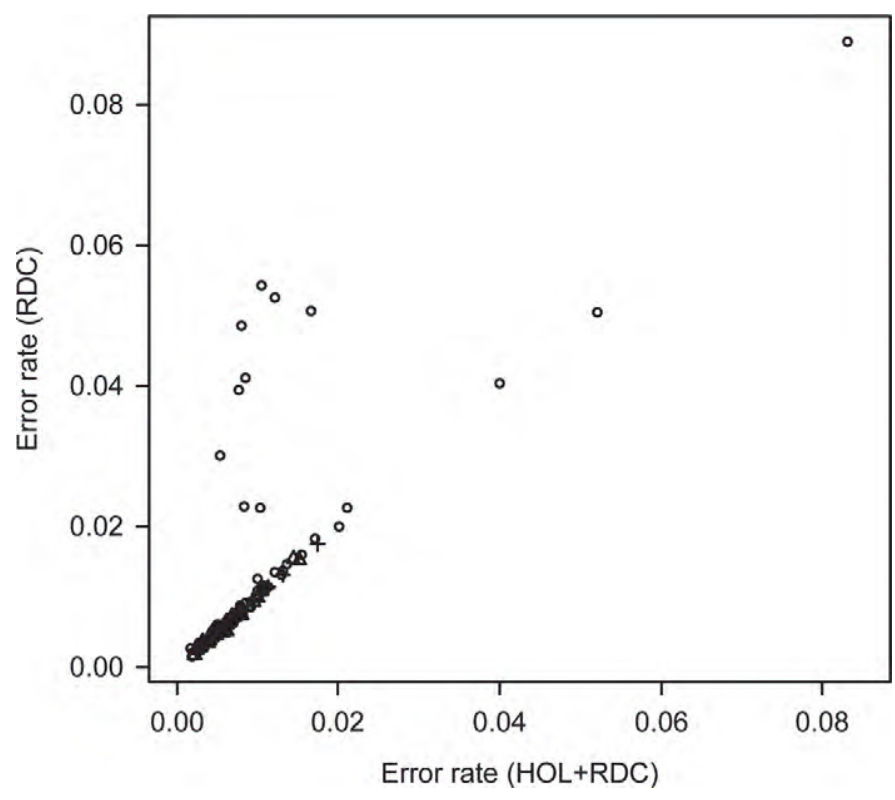

Figure 3. Error rates per animal using a pure Nordic Red (RDC) reference versus a Holstein (HOL) + RDC reference for Danish (circles), Finnish (triangles), and Swedish (plus signs) animals.

tigate whether the combined RDC-Holstein reference improved imputation accuracy when only minor alleles were considered (alleles with a frequency of less than $0.5)$, the imputation error rate based on minor alleles was calculated. As shown in the right panel of Figure 4, small gains in accuracy were still seen on average when

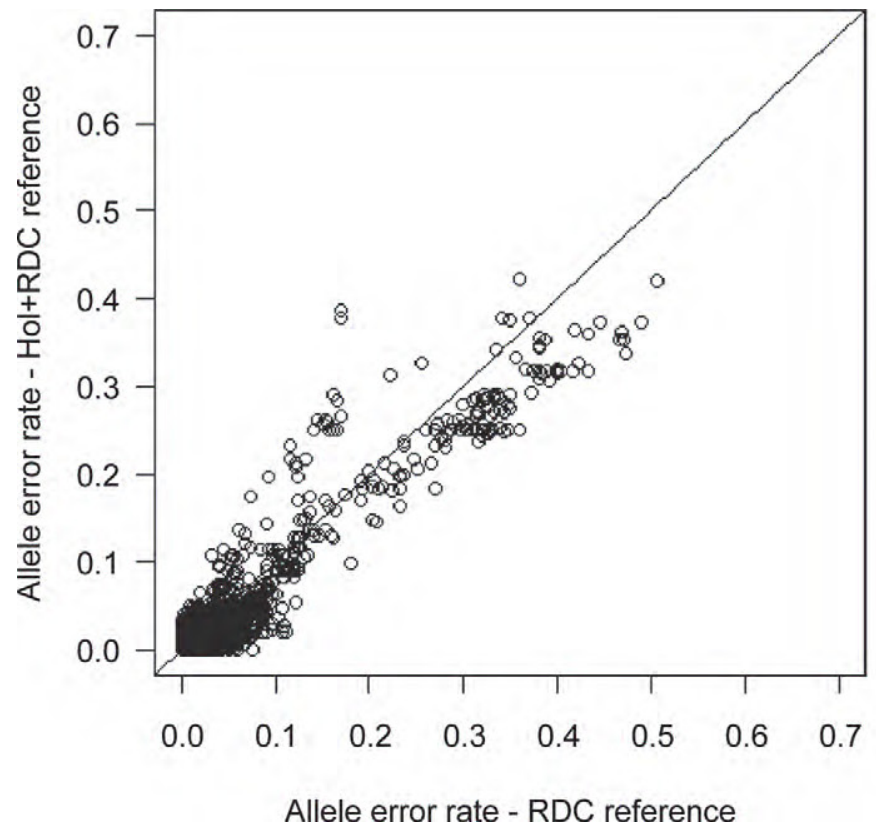

using the combined RDC-Holstein reference. However, some rare alleles that were imputed correctly when using only the RDC reference were incorrectly imputed with the inclusion of Holstein in the reference. This was likely caused by differences in allele frequencies between the breeds in the reference, which would change the probability of imputing the alleles.

Summarizing the imputation accuracy in intervals of minor allele frequency as shown in Figure 5 gave a clearer pattern than is shown in Figure 4. For both the entire RDC validation group and only Danish Red, increases in both the allele correct rate $(1$ - error rate) and the correlation between true and imputed genotypes were seen for all bins when Holstein animals were included in the imputation reference. For Swedish Red and Finnish Ayrshires, however, very little difference was seen in the 2 scenarios, and a small tendency existed for the imputation accuracy to decrease in bins with a low minor allele frequency when Holstein animals were included in the reference. Figure 4 also shows that using the allele error rate as a measure of imputation accuracy hid the difficulty of imputing rare alleles, whereas the correlation between true and imputed genotypes clearly decreased for the markers with low minor allele frequency values. A similar trend was found in Hickey et al. (2012).

Results in this study are in line with similar studies on imputation from $50 \mathrm{~K}$ to HD marker data in dairy cattle. For Australian Holsteins, Erbe et al. (2012) found that the percentage of correctly imputed

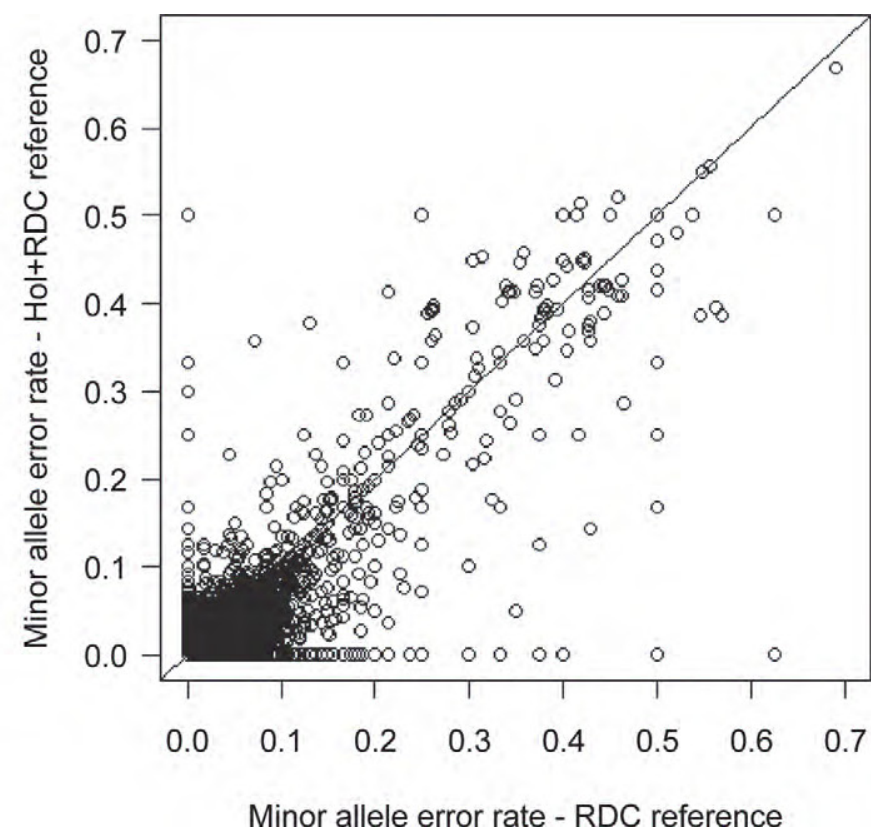

Figure 4. Mean error rates for each locus on chromosome 1 for Nordic Red (RDC) validation animals with an RDC reference versus a Holstein (HOL) + RDC reference, and error rates for the minor allele at each locus on chromosome 1 for all RDC validation animals with the $\mathrm{RDC}$ reference compared with the HOL + RDC reference. 
RDC
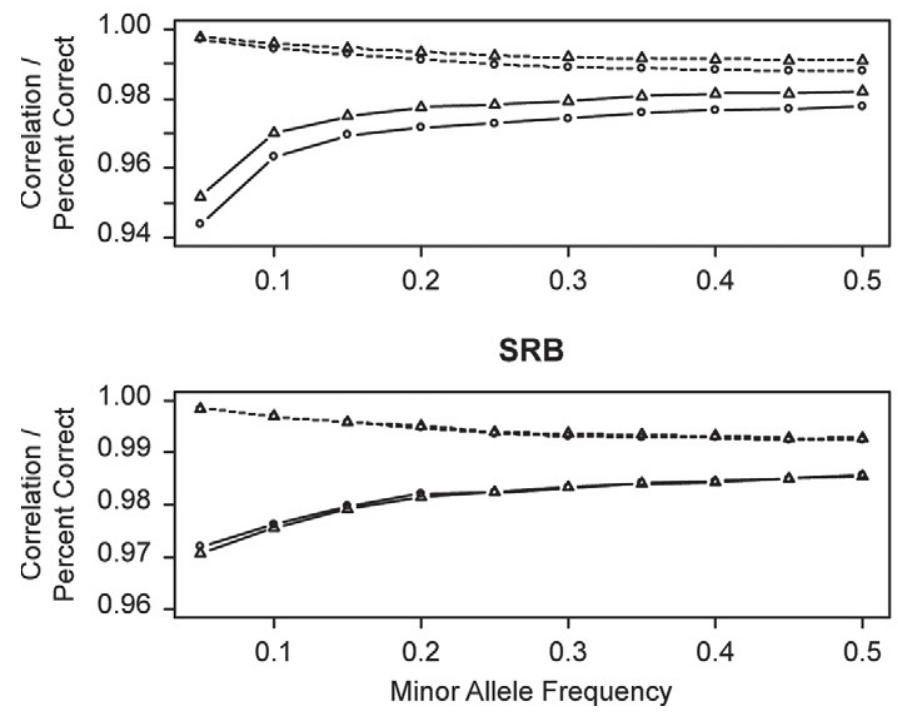

RDM

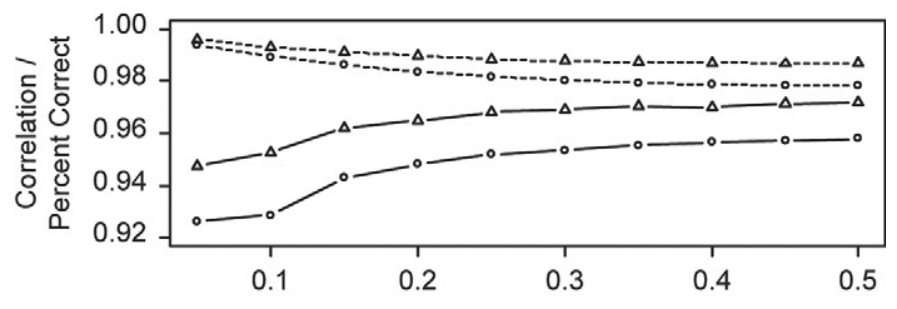

FAY

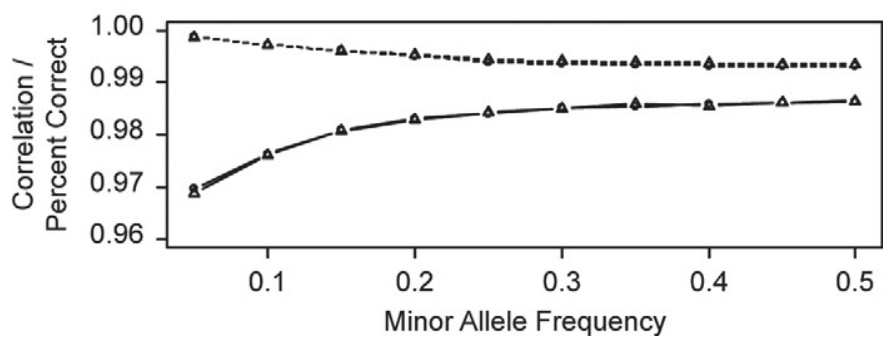

Figure 5. Allele correct rate (dashed lines) and correlation between true and imputed genotypes (solid lines) for all Nordic Red (RDC), Danish Red (RDM), Swedish Red (SRB), or Finnish Ayrshire (FAY) validation animals using an RDC reference (circles) or an RDC + Holstein reference (triangles). Imputation accuracy was calculated in intervals of $5 \%$ of the minor allele frequency.

genotypes was $98 \%$, which corresponded to an allele error rate of about $1 \%$. Accuracies found here were higher than those found when imputing from $3 \mathrm{~K}$ to $50 \mathrm{~K}$ marker panels in dairy cattle (Weigel et al., 2010; Dassonneville et al., 2011b; Mulder et al., 2012). This increase in accuracy was caused by the stronger LD on the marker panel for the validation animals ( $50 \mathrm{~K}$ vs. $3 \mathrm{~K} / 6 \mathrm{~K})$ as well as the stronger $\mathrm{LD}$ in the reference.

In summary, results from the study showed that when imputing from a 50K SNP marker panel to an HD SNP marker panel in the RDC populations, using a combined RDC reference gave a higher accuracy of imputation than using single-nationality references. Adding Holstein bulls to the reference led to further improvement of imputation for Danish Red animals, but not for Swedish and Finnish animals. This indicates that the multibreed reference is helpful when genetic links between the breeds are strong.

\section{REFERENCES}

Brøndum, R. F., E. Rius-Vilarrasa, I. Stranden, G. Su, B. Guldbrandtsen, W. F. Fikse, and M. S. Lund. 2011. Reliabilities of genomic prediction using combined reference data of the Nordic Red dairy cattle populations. J. Dairy Sci. 94:4700-4707.

Browning, B. L., and S. R. Browning. 2009. A unified approach to genotype imputation and haplotype-phase inference for large data sets of trios and unrelated individuals. Am. J. Hum. Genet. 84:210-223.

Dassonneville, R., R. F. Brondum, T. Druet, S. Fritz, F. Guillaume, B. Guldbrandtsen, M. S. Lund, V. Ducrocq, and G. Su. 2011a. Effect of imputing markers from a low-density chip on the reliability of genomic breeding values in Holstein populations. J. Dairy Sci. 94:3679-3686.
Dassonneville, R., R. F. Brøndum, T. Druet, S. Fritz, F. Guillaume, B. Guldbrandtsen, M. S. Lund, V. Ducrocq, and G. Su. 2011b. Effect of imputing markers from a low-density chip on the reliability of genomic breeding values in Holstein populations. J. Dairy Sci. 94:3679-3686.

Erbe, M., B. J. Hayes, L. K. Matukumalli, S. Goswami, P. J. Bowman, C. M. Reich, B. A. Mason, and M. E. Goddard. 2012. Improving accuracy of genomic predictions within and between dairy cattle breeds with imputed high density SNP panels. J. Dairy Sci. 95:4114-4129.

Hayes, B. J., P. J. Bowman, H. D. Daetwyler, J. W. Kijas, and J. H. J. van der Werf. 2012. Accuracy of genotype imputation in sheep breeds. Anim. Genet. 43:72-80.

Hickey, J. M., J. Crossa, R. Babu, and G. de los Campos. 2012. Factors affecting the accuracy of genotype imputation in populations from several maize breeding programs. Crop Sci. 52:654-663.

Huang, L., Y. Li, A. B. Singleton, J. A. Hardy, G. Abecasis, N. A. Rosenberg, and P. Scheet. 2009a. Genotype-imputation accuracy across worldwide human populations. Am. J. Hum. Genet. $84: 235-250$.

Huang, L., C. L. Wang, and N. A. Rosenberg. 2009b. The relationship between imputation error and statistical power in genetic association studies in diverse populations. Am. J. Hum. Genet. 85:692-698.

Lund, M. S., A. P. De Roos, A. G. de Vries, T. Druet, V. Ducrocq, S. Fritz, F. Guillaume, B. Guldbrandtsen, Z. Liu, R. Reents, C. Schrooten, F. Seefried, and G. Su. 2011. A common reference population from four European Holstein populations increases reliability of genomic predictions. Genet. Sel. Evol. 43:43.

Mulder, H. A., M. P. L. Calus, T. Druet, and C. Schrooten. 2012. Imputation of genotypes with low-density chips and its effect on reliability of direct genomic values in Dutch Holstein cattle. J. Dairy Sci. 95:876-889.

Weigel, K. A., G. de los Campos, A. I. Vazquez, G. J. M. Rosa, D. Gianola, and C. P. Van Tassell. 2010. Accuracy of direct genomic values derived from imputed single nucleotide polymorphism genotypes in Jersey cattle. J. Dairy Sci. 93:5423-5435.

Zhang, Z., and T. Druet. 2010. Marker imputation with low-density marker panels in Dutch Holstein cattle. J. Dairy Sci. 93:54875494. 